\title{
Detector evaporativo com espalhamento de luz: princípios de operação e aplicações em cromatografia líquida de alta eficiência
}

\author{
Mário Henrique Gonzalez, Silmara Rossana Bianchi, \\ Catarinie Diniz Pereira, Neila Maria Cassiano, Quezia Bezerra Cass* \\ Departamento de Química, Universidade Federal de São Carlos - UFSCar, \\ CP 676, Cep 13565-970, São Carlos, SP, Brasil \\ e-mail:quezia@pq.cnpq.br
}

Resumo

O detector evaporativo com espalhamento de luz (ELSD) é um detector semiuniversal para cromatografia líquida de alta eficiência (HPLC). Esse detector possibilita a análise de compostos menos voláteis que a fase móvel, independente de suas estruturas químicas, e é uma importante ferramenta para determinar analitos sem cromóforos ou que tenham somente grupos cromóforos fracos. Este artigo apresenta os princípios de operação do ELSD e vários exemplos de aplicação em diferentes matrizes.

Palavras-chave

Detector evaporativo com espalhamento de luz; HPLC; aplicações.

\section{Evaporative light scattering detector: operation principles and applications in high performance liquid chromatography}

\section{Abstract}

The evaporative light scattering detector (ELSD) is a semi-universal detector for high performance liquid chromatography (HPLC). This detector allows analysis of compounds less volatiles than the mobile phase, regardless of their chemical structure, and it is a valuable tool to determine analytes without chromophores or that have only weak chromophores groups. This paper present the operation principles of ELSD and several examples of applications in different matrixes are given.

Keywords

Evaporative light scattering detector; HPLC; applications. 


\section{Introdução}

O sistema de detecção baseado na absorção de radiação eletromagnética nas regiões do ultravioleta/visível (UV-Vis) é ainda hoje o mais empregado no Brasil em cromatografia líquida de alta eficiência (HPLC, High Performance Liquid Chromatography). Esse detector óptico apresenta boa sensibilidade, ampla faixa linear, baixo limite de detecção e estabilidade frente a mudanças na composição da fase móvel. Para a sua utilização, entretanto, é necessário que os analitos tenham cromóforos que absorvam na região do UV-Vis ${ }^{[1]}$.

Alternativamente, o detector de índice de refração (RID, Refractive Index Detector) e o espectrômetro de massas (MS, Mass Spectrometry) podem ser empregados na análise de importantes classes de compostos que não possuem cromóforos ou que tenham somente grupos cromóforos fracos como, por exemplo, carboidratos, lipídeos, saponinas e alcaloides. No entanto, o RID é muito sensível às variações de temperatura e à composição da fase móvel, o que o torna inadequado para uso em eluição por gradiente, enquanto o MS, apesar da alta sensibilidade e seletividade, tem um elevado custo para aquisição e manutenção do equipamento ${ }^{[1]}$.

No fim da década de 70, o detector evaporativo com espalhamento de luz (ELSD, Evaporative Light Scattering Detector) foi introduzido no campo da análise cromatográfica ${ }^{[2]}$ e desde a comercialização do primeiro equipamento, no início dos anos 80 , o número de trabalhos publicados que descreve o uso deste detector em HPLC tem aumentado anualmente. O ELSD possibilita a análise de compostos menos voláteis que a fase móvel, independente de suas estruturas químicas, e o uso de eluição por gradiente ${ }^{[1,3]}$.
Em 2005, Ganzera e Stuppner ${ }^{[4]}$ revisaram o uso do ELSD na análise de produtos naturais e Megoulas e Koupparis ${ }^{[5]}$ descreveram o uso operacional, vantagens/limitações e aplicações desse detector em cromatografia líquida e com fluido supercrítico.

O presente artigo discute os princípios de operação do ELSD e destaca relevantes trabalhos publicados no período de 2005 a 2011 nas análises de carboidratos e glicosídeos, lipídeos, alcaloides, saponinas e terpenoides em diferentes matrizes.

\section{Princípios de operação do ELSD}

O princípio de operação do ELSD envolve três processos consecutivos, os quais estão interrelacionados e influenciam ou são influenciados pelos outros processos: nebulização do eluente da coluna cromatográfica, evaporação da fase móvel e medida da intensidade de luz espalhada pelas moléculas do analito que atravessam um feixe de radiação eletromagnética e geram um sinal proporcional ao número de fótons espalha$\operatorname{dos}(\operatorname{detec} c ̧ a ̃ o))^{[5,6]}$.

\subsection{Etapa de nebulização}

Nessa etapa inicial, o eluente que sai da coluna cromatográfica entra em um nebulizador onde é convertido em uma névoa gasosa (aerossol) por um fluxo de gás carreador, usualmente nitrogênio ou ar comprimido, em alta velocidade. A vazão do gás carreador deve ser ajustada em relação à vazão da fase móvel com o intuito de se obter gotículas com tamanho adequado para assegurar uma boa sensibilidade na deteç̧ão ${ }^{[5,6]}$.

Em alguns modelos de ELSD comercialmente disponíveis, o eluente nebulizado entra em um tubo aquecido onde acontece a evaporação da fase móvel. Outros modelos, no entanto, 
incorporam uma câmara de nebulização, cuja finalidade é remover as gotas com diâmetros maiores $^{[3,5]}$. Cada tipo de detector apresenta características próprias e as vantagens/limitações de cada equipamento dependem do tipo de análise a ser realizada ${ }^{[5]}$.

Algumas propriedades físico-químicas da fase móvel, tais como densidade, viscosidade e tensão superficial também influenciam diretamente o tamanho das gotículas do aerossol ${ }^{[3,5]}$. A equação empírica a partir da qual é possível relacionar o diâmetro médio das gotículas formadas no processo de nebulização $\left(\mathrm{D}_{0}\right)$ com as propriedades da fase móvel, a vazão/velocidade do gás carreador e a da fase móvel é dada por ${ }^{[6]}$ :

$$
D_{0}=\frac{585 \sqrt{\sigma}}{\left(v_{g}-v_{1}\right) \sqrt{\rho}}+597\left(\frac{\mu}{\sqrt{\sigma \rho}}\right)^{0,45}\left(\frac{1000 Q_{1}}{Q_{g}}\right)^{1,5}
$$

onde $\sigma, \rho$ e $\mu$ são, respectivamente, tensão superficial, densidade e viscosidade da fase móvel, $\left(v_{g}-v_{1}\right)$ é a diferença entre as velocidades do gás carreador e da fase móvel e $\mathrm{Q}_{\mathrm{l}} / \mathrm{Q}_{\mathrm{g}}$ é a razão entre as vazões da fase móvel e do gás carreador.

A partir da Equação 1, aplicável para gotículas com tamanhos na faixa de 15-90 $\mu \mathrm{m}$ e densidade da fase móvel entre 0,7 e 1,2 g.mL $\mathrm{mL}^{-1[6,7]}$, é possível observar que uma diminuição na velocidade do gás carreador $\left(v_{g}\right)$, mantendo constante ou aumentando a velocidade da fase móvel $\left(v_{1}\right)$, resulta em um aumento no diâmetro médio das gotículas no aerossol, o que propicia uma maior sensibilidade do detector. No entanto, se a velocidade do gás carreador for muito baixa, a fase móvel pode não ser totalmente nebulizada e/ou posteriormente evaporada, resultando em um aumento no nível de ruídos na linha de base do cromatograma $^{[5]}$.

\subsection{Ełapa de evaporação}

O aerossol formado na etapa de nebulização é direcionado para um tubo aquecido onde a fase móvel é evaporada, restando apenas partículas secas do analito. A temperatura de aquecimento do tubo deve ser ajustada em função da composição da fase móvel e propriedades do analito para que a fase móvel seja completamente removida sem que haja perdas do composto por evaporação ou decomposição térmica. $\mathrm{O}$ uso de baixas temperaturas favorece a formação de partículas maiores e mais uniformes, o que aumenta a sensibilidade do detector. No entanto, tais condições de temperatura podem propiciar uma evaporação da fase móvel incompleta, ocasionando um excessivo nível de ruídos na linha de base do cromatograma. Por outro lado, temperaturas excessivamente altas podem levar a uma perda de sensibilidade na detecção devido à sublimação do analito ${ }^{[3,5]}$.

Após a completa evaporação da fase móvel, o diâmetro das partículas do analito (d) pode ser estimado a partir da Equação $2^{[7]}$ :

$$
d=D_{0}\left(\frac{C}{\rho_{a}}\right)^{1 / 3}
$$

A resposta do detector é influenciada pelo tamanho das partículas do analito (d) que, por sua vez, é dependente do diâmetro médio das gotículas do aerossol formadas no processo de nebulização $\left(\mathrm{D}_{0}\right)$ e da concentração $(\mathrm{C})$ e densidade do analito $\left(\rho_{\mathrm{a}}\right)$.

\subsection{Etapa de detecção}

Após a etapa de evaporação, as partículas do analito (d) são transportadas, pelo gás carreador, para uma cela óptica, atravessam um feixe de radiação eletromagnética e a luz espalhada é medida por um fotodiodo ou um fotomultiplicador. O tipo de interação das partículas do ana- 
lito com a radiação eletromagnética depende do tamanho, forma e propriedades da superfície das partículas formadas nos processos de nebulização e evaporação, assim como do comprimento de onda da luz incidente ${ }^{[3,5]}$.

As principais interações que governam o espalhamento de luz no ELSD são dispersão de Rayleigh, dispersão de Mie e refração/reflexão, sendo que a relevância de cada uma delas está relacionada com o diâmetro das partículas (d) que entram na cela de detecção e com o comprimento de onda $(\lambda)$ do feixe de radiação da luz incidente. Para $d / \lambda<0,1$ prevalece a teoria de Rayleigh, para $0,1<\mathrm{d} / \lambda<2$ haverá predominância da teoria de Mie e com $\mathrm{d} / \lambda>2$ ocorre refração/reflexão ${ }^{[3]}$.

Devido à natureza das interações entre as partículas do analito com a radiação eletromagnética, a resposta do detector é relacionada com a massa do analito pela Equação 3:

$$
A=a M^{b}
$$

onde $\mathrm{A}$ é área do pico cromatográfico e $\mathrm{M}$ é a massa do analito injetado. As constantes a e b são determinadas pelas condições cromatográficas (vazões do gás carreador e da fase móvel, temperatura de evaporação, propriedades físico-químicas da fase móvel, etc.), características e concentração do analito ${ }^{[5,7]}$.

Para a obtenção de curvas analíticas é necessário o uso de coordenadas logarítmicas a partir da Equação 4:

$$
\log A=\log a+b \log M
$$

Uma interessante aplicação do ELSD em análises quantitativas sem o uso de padrões de referência dos analitos foi realizada por Megoulas e Koupparis ${ }^{[8]}$. Considerando o fato de o ELSD apresentar fatores de resposta equivalentes para moléculas com fórmulas estruturais semelhantes e massas moleculares próximas e a falta de padrões de referência para as quatro substâncias análogas que compõem a gentamicina, designadas como $\mathrm{C}_{1}, \mathrm{C}_{1 \mathrm{a}}, \mathrm{C}_{2}$ e $\mathrm{C}_{2 \mathrm{a}}$, os autores desenvolveram um método para determinação desses compostos em formulações farmacêuticas usando canamicina A, um composto estruturalmente semelhante à gentamicina, como padrão externo secundário. Assumindo que os coeficientes linear e angular das curvas analíticas logarítmicas dos componentes da gentamicina e da canamicina $\mathrm{A}$ são estatisticamente iguais e levando em consideração as proporções de cada componente foi possível quantificar gentamicina $C_{1}$, gentamicina $\mathrm{C}_{1 \mathrm{a}}$, gentamicina $\mathrm{C}_{2}$ e gentamicina $\mathrm{C}_{2 \mathrm{a}}$ em produtos comerciais.

A Figura 1 ilustra de forma esquemática os processos de nebulização, evaporação e detecção que ocorrem sucessivamente em um ELSD.

\section{Aplicações analíticas}

\subsection{Análise de carboidratos e glicosídeos}

Carboidratos são substâncias poli-hidroxiladas de aldeídos, cetonas, alcoóis, ácidos e seus derivados simples, bem como seus polímeros que tenham ligações poliméricas do tipo acetal ${ }^{[9]}$.

(2)

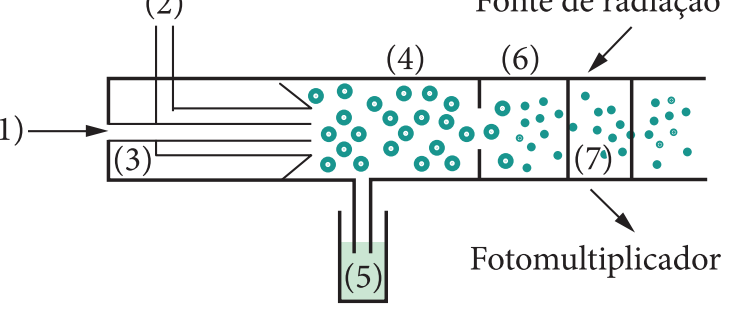

Figura 1 Representação esquemática de um detector evaporativo com espalhamento de luz $(E L S D) ; 1)$ elvente cromatográfico; 2) gás carreador; 3) nebulizador; 4) câmara de nebulização; 5) dreno; 6) tubo de aquecimento e 7) cela óptica. Adaptada da referência 5 . 
Uma importante família de biomoléculas pertencentes ao grupo dos carboidratos são os antibióticos aminoglicosídeos. Tais compostos, usados no tratamento de doenças em seres humanos e animais causadas por bactérias aeróbicas gram-positivas e gram-negativas, são caracterizados pela presença de dois ou mais amino-açúcares ligados a um anel aminociclitol (um hidroxicicloexano amino substituído) por ligações glicosídicas ${ }^{[10]}$.

Como os carboidratos e aminoglicosídeos não possuem grupos cromóforos em suas estruturas químicas, alguns métodos de análise por HPLC fazem uso de derivação da amostra com detecção no UV/vis ou por fluorescência. No entanto, técnicas de derivação podem apresentar uma série de problemas decorrentes das condições experimentais como, por exemplo, reação incompleta, formação de subprodutos, longo tempo de preparo da amostra, entre outros. Uma alternativa aos métodos de derivação é a análise desses compostos utilizando o ELSD. Exemplos da aplicação do ELSD nas análises de carboidratos e aminoglicosídeos em diferentes matrizes são apresentados na Tabela 1.

\subsection{Análise de lipídeos}

A família dos lipídeos é constituída por uma ampla gama de compostos com diferentes estruturas químicas (ácidos graxos livres, triacilgliceróis, fosfolipídeos, esfingolipídeos, esteroides, etc.) que desempenham uma série de funções nos sistemas biológicos, tais como armazenar energia, ser componente das membranas celulares, precursores na biossíntese de importantes compostos químicos e de reguladores do metabolismo celular ${ }^{[22]}$.

Tabela 1 Uso do ELSD na análise de carboidratos e aminoglicosídeos em diferentes matrizes.

\begin{tabular}{|c|c|c|c|}
\hline Analito & Matriz & LD ou LQ & Ref. \\
\hline $\begin{array}{l}\text { Glicose, frutose, maltose, } \\
\text { maltotriose e maltotetrose }\end{array}$ & Cerveja & LD: $0,005-0,01 \mathrm{~g} \cdot \mathrm{L}^{-1}$ & 11 \\
\hline $\begin{array}{l}\text { Glicose, frutose, sacarose, xilose, } \\
\text { maltose e rafinose }\end{array}$ & Folha de tabaco & LD: $2-4 \mu \mathrm{g} \cdot \mathrm{mL}^{-1}$ & 12 \\
\hline $\begin{array}{c}\text { Glicose, arabinose, xilose, ramanose, } \\
\text { sacarose, xilitol, manitol, celobiose, } \\
\text { lactose e inositol }\end{array}$ & $\begin{array}{l}\text { Planta da espécie: } \\
\text { Tinospora cordifolia }\end{array}$ & $\mathrm{LQ:}: 25,23-134,2 \mu \mathrm{g} \cdot \mathrm{mL}^{-1}$ & 13 \\
\hline \multirow{2}{*}{ Neomicina } & $\mathrm{FF}$ & LD: $1,0 \mu \mathrm{g} \cdot \mathrm{mL}^{-1}$ & 14 \\
\hline & $\mathrm{FF}^{(\mathrm{a})}$ & $\mathrm{LD}: 0,60 \mu \mathrm{g} \cdot \mathrm{mL}^{-1}$ & 15 \\
\hline Tobramicina & $\mathrm{FF}$ & $(*)$ & 16 \\
\hline Canamicina a (1) e canamicina b (2) & $\begin{array}{l}\text { MP, FF e meio de } \\
\text { cultura bacteriano }\end{array}$ & $\begin{array}{c}\mathrm{LD}: 0,20 \mu \mathrm{g} \cdot \mathrm{mL}^{-1}(1) \mathrm{e} \\
1,4 \mu \mathrm{g} \cdot \mathrm{mL}^{-1}(2)\end{array}$ & 17 \\
\hline Espectinomicina & MP & $\mathrm{LD}: 6,0 \mu \mathrm{g} \cdot \mathrm{mL}^{-1}$ & 18 \\
\hline Amicacina (1) e canamicina (2) & $M P$ e FF & $\begin{array}{l}\mathrm{LD}: 2,2 \mu \mathrm{g} \cdot \mathrm{mL}^{-1}(1) \mathrm{e} \\
2,5 \mu \mathrm{g} \cdot \mathrm{mL}^{-1}(2)\end{array}$ & 19 \\
\hline $\begin{array}{c}\text { Estreptomicina (1) e } \\
\text { diidroestreptomicina (2) }\end{array}$ & $\begin{array}{l}\text { MP, FF e meio de } \\
\text { cultura bacteriano }\end{array}$ & $\begin{array}{l}\text { LD: } 0,7 \mu \mathrm{g} \cdot \mathrm{mL}^{-1}(1) \mathrm{e} \\
\quad 0,5 \mu \mathrm{g} \cdot \mathrm{mL}^{-1}(2)\end{array}$ & 20 \\
\hline Arbecacina & FF & LD: $4,5 \mu \mathrm{g} \cdot \mathrm{mL}^{-1}$ & 21 \\
\hline
\end{tabular}

LD: limite de detecção; LQ: limite de quantificação; FF: formulações farmacêuticas; MP: matéria-prima; ${ }^{(a)}$ : neomicina em associação com bacitracina. $\left({ }^{*}\right)$ : não informado. 
Há um grande interesse na análise de lipídeos em alimentos. O desenvolvimento de métodos para identificação/quantificação de triacilgliceróis, por exemplo, é de suma importância para confirmar a autenticidade, detectar adulterações e definir a composição química de óleos vegetais e gorduras ${ }^{[23]}$. Os fosfolipídeos e esfingolipídeos são responsáveis pelo sabor, textura e propriedades nutricionais de carnes, leite e seus derivados.

Na última década, a cromatografia líquida de alta eficiência acoplada ao detector de espalhamento de luz (HPLC-ELSD) consolidou-se como uma importante ferramenta analítica para a determinação de lipídeos em diferentes tipos de amostras (Tabela 2).

\subsection{Análise de alcaloides}

Veratrum nigrum L. e Veratrum dahuricum, duas espécies de plantas herbáceas pertencentes ao gênero Veratrum (Liliaceae), são amplamente empregadas na medicina tradicional chinesa para o tratamento de hipertensão, afasia, epilepsia, disenteria, malária, dentre outras enfermidades. Os componentes bioativos responsáveis pelas atividades farmacológicas e efeitos tóxicos dessas plantas são alcaloides esteroidais ${ }^{[31-33]}$.
Cong et al. ${ }^{[31]}$ desenvolveram um método, por HPLC-ELSD, para análise de dez alcaloides esteroidais, escolhidos como marcadores químicos, em extratos aquosos de raízes e rizomas de Veratrum nigrum L. Todos os compostos foram eluídos com separação em linha de base em 78 min, no modo de eluição gradiente, com fase móvel constituída por acetonitrila e solução de trietilamina $0,03 \%(\mathrm{v} / \mathrm{v})$. Os resultados mostraram que a concentração dos alcaloides é dependente do procedimento de preparo dos extratos.

Wang et al. ${ }^{[32]}$ desenvolveram um método analítico de perfil cromatográfico (fingerprint) utilizando HPLC acoplada ao ELSD, para o controle de qualidade químico de Veratrum nigrum $\mathrm{L}$. Três alcalóides foram quantificados em raízes e partes aéreas da planta, obtidas a partir de dez lotes de Veratrum nigrum L. coletados em diferentes locais, para avaliar a uniformidade lote a lote das amostras.

Tang et al. ${ }^{[33]}$ realizaram um estudo para determinar o teor de seis alcaloides esteroidais em extratos metanólicos de oito lotes de Veratrum dahuricum coletados em diferentes estações do ano. As técnicas cromatográficas HPLC-MS $^{\mathrm{n}}$ e HPLC-ELSD foram usadas, respectivamente, para identificação estrutural e quantificação dos constituintes químicos. Os resultados

Tabela 2 Uso do ELSD na análise de lipídeos em diferentes matrizes.

\begin{tabular}{cccc}
\hline Analito & Matriz & LD ou LQ & Ref. \\
\hline Triacilgliceróis & Óleos vegetais & LQ: $0,15-1,2 \mu \mathrm{g} \cdot \mathrm{mL}^{-1}$ & 23 \\
\cline { 2 - 3 } Triacilgliceróis e ácidos graxos & Óleos de girassol/soia & 24 \\
\cline { 2 - 3 } Fosfolipídeos & Carne de pato & LQ: $0,13-0,15 \mu \mathrm{g} \cdot \mathrm{mL}^{-1}$ & 25 \\
Esfingolipídeos & Leite & LQ: $0,91-2,28 \mathrm{~g}$ & 26 \\
\cline { 2 - 4 } Diversas classes de lipídeos & Peixes & LD $<1 \mu \mathrm{g}$ & 27 \\
Fitosteróis & Zooplâncton marinho & $(*)$ & 28 \\
\cline { 2 - 4 } & FF & LQ: $5 \mu \mathrm{g} \cdot \mathrm{mL}^{-1}$ & 29 \\
\hline
\end{tabular}

LD: limite de detecção; LQ: limite de quantificação; FF: formulações farmacêuticas; (*): não informado. 
demonstraram que as concentrações dos alcaloides nas plantas variaram de forma significativa dependendo da época de coleta, confirmando a necessidade de um controle de qualidade da matéria-prima vegetal.

\subsection{Análise de saponinas e terpenoides}

Saponinas são moléculas com estruturas complexas constituídas por unidades de açúcares covalentemente ligadas a um esqueleto triterpênico ou esteroidal. Esse grupo de metabólitos secundários são encontrados em uma grande variedade de espécies de plantas ${ }^{[34]}$.

O ginseng, uma erva medicinal utilizada na medicina tradicional dos países asiáticos há mais de 2000 anos, é derivado das raízes e rizomas de várias espécies de plantas do gênero Panax $(\text { Araliaceae })^{[35]}$. Os principais componentes bioativos do ginseng são saponinas triterpênicas, também chamadas de ginsenosídeos, às quais são atribuídas propriedades farmacológicas que incluem efeitos neuroprotetores, imunoestimulantes e antioxidantes, dentre outros ${ }^{[36]}$.

Saponinas triterpênicas e esteroidais também são responsáveis pelos efeitos farmacológicos de outras espécies vegetais de uso corrente na medicina popular asiática: Pulsatilla koreana (Ranunculaceae), Bupleurum falcatrum L. (Umbelliferae), Clematis spp. (Ranunculaceae), Paris spp. e Trillium tschonoskii (Trilliaceae) ${ }^{[37-41]}$.

Ginkgo biloba L. (Ginkgoaceae) é uma das espécies de plantas mais antigas do mundo. A principal indicação das preparações à base de Ginkgo é o tratamento de problemas ligados à insuficiência vascular cerebral e periférica, sendo sua eficácia clínica atribuída às trilactonas terpênicas (gincolídeos A, B, C e J e bilobalídeo) e flavonoides. Os gincolídeos (diterpenos) inibem o fator de agregação plaquetária, o bilobalídeo (lac- tona terpênica) apresenta propriedades neuroprotetoras, enquanto os flavonoides atuam como captores de radicais livres. Os extratos padronizados de Ginkgo biloba, preparados a partir das folhas secas, são empregados na fabricação de medicamentos fitoterápicos. Para assegurar o controle de qualidade e a ação farmacológica dos fitomedicamentos, os extratos são produzidos de forma a garantir uma concentração definida das substâncias específicas ou grupos de compostos com eficácia terapêutica conhecida ${ }^{[42]}$.

Outras espécies de plantas são fontes de terpenoides dotados de importantes ações farmacológicas. A Artemisia annua L., originária das regiões de clima temperado da Ásia, é utilizada na medicina tradicional chinesa há vários séculos para o tratamento da malária. A artemisinina, uma lactona sesquiterpênica, é o princípio ativo responsável pela grande atividade contra Plasmodium falciparum e Plasmodium vivax, os principais parasitas causadores dessa doença ${ }^{[43]}$.

A Tabela 3 apresenta exemplos do uso de HPLC-ELSD na análise de saponinas terpênicas/ esteroidais e terpenoides aplicadas no controle de qualidade de matéria-prima vegetal, extratos de plantas e preparações farmacêuticas.

\subsection{Acoplamento HPLC-DAD-ELSD}

$\mathrm{O}$ acoplamento on-line do equipamento de HPLC aos detectores por arranjo de diodos (DAD, Diode Array Detector) e ELSD tem sido utilizado com sucesso na determinação simultânea de multicomponentes estruturalmente diferentes em matrizes complexas. Alguns exemplos da aplicação do sistema HPLC-DAD-ELSD são descritos a seguir.

Wang et al. ${ }^{[50]}$ desenvolveram um método para análise de oito nucleosídeos e nucleobases (monitorados a $254 \mathrm{~nm}$ ), três carboidratos 
Tabela 3 Uso do ELSD na análise de saponinas e terpenoides em diferentes matrizes.

\begin{tabular}{|c|c|c|c|}
\hline Analito & Matriz & LD ou LQ & Ref. \\
\hline \multirow{4}{*}{$\begin{array}{l}\text { Saponinas } \\
\text { triterpênicas }\end{array}$} & Ginseng (produto acabado) & LQ: $37-186$ ng & 36 \\
\hline & Raíz de Pulsatilla koreana & $\mathrm{LQ}<33,8 \mu \mathrm{g} \cdot \mathrm{mL}^{-1}$ & 37 \\
\hline & Raíz de Bupleurum falcatum L. & $\mathrm{LQ}: 2,0-6,0 \mu \mathrm{g} \cdot \mathrm{mL}^{-1}$ & 38 \\
\hline & Raíz de Clematis spp. & LD: 0,169 - 0,506 $\mu \mathrm{g}$ & 39 \\
\hline \multirow{3}{*}{$\begin{array}{l}\text { Saponinas } \\
\text { esteroidais }\end{array}$} & Raízes de Paris spp. e Trillium tschonoskii & LQ: $189,0-400,2$ ng & 40 \\
\hline & Cápsulas de Gongxuening & LQ: $0,27-0,76 \mu \mathrm{g}$ & 41 \\
\hline & Extrato de Tripterygium wilfordii & $\mathrm{LQ}<0,297 \mu \mathrm{g}$ & 44 \\
\hline Terpenoides & $\begin{array}{l}\text { Diferentes partes das plantas Ilex hainanensis, } \\
\text { I. stewardii e I. pubescens }\end{array}$ & $\mathrm{LQ}: 20,9-36,8 \mu \mathrm{g} \cdot \mathrm{mL}^{-1}$ & 45 \\
\hline \multirow{2}{*}{$\begin{array}{l}\text { Gincolídeos e } \\
\text { bilobalídeos }\end{array}$} & Extrato bruto de Ginkgo biloba & LQ: $825-1320 \mu \mathrm{g} \cdot \mathrm{mL}^{-1}$ & 46 \\
\hline & Cápsulas/comprimidos de G. biloba & LQ: $62,50 \mathrm{ng}$ & 47 \\
\hline \multirow{2}{*}{ Artemisinina } & Extrato de Artemisinin annua & LQ: $50-250 \mu \mathrm{g} \cdot \mathrm{mL}^{-1}$ & 48 \\
\hline & Biomassa e extratos de $A$. annua & LQ: $0,10 \mathrm{mg} \cdot \mathrm{mL}^{-1}$ & 49 \\
\hline
\end{tabular}

LD: limite de detecção; LQ: limite de quantificação.

e um aminoácido (detectados com o ELSD) em diferentes amostras de Cordyceps, um compósito de estromas do fungo Cordyceps sinensis. Os resultados mostraram que os carboidratos podem ser usados como marcadores químicos na discriminação de diferentes espécies de Cordyceps.

Avula et al. ${ }^{[51]}$ realizaram a determinação de quatro flavonoides e quatro triterpenoides em extratos/produtos relacionados de Sutherlandia Frutescens (Fabaceae), uma planta medicinal usada no tratamento de problemas estomacais, diabetes e vários tipos de inflamações em alguns países da África. O comprimento de onda usado na quantificação dos flavonoides foi $260 \mathrm{~nm}$, enquanto os triterpenoides foram determinados com o ELSD.

Além dos dois trabalhos citados, outros foram realizados utilizando o acoplamento HPLC-DAD-ELSD para quantificação de diversos constituintes bioativos pertencentes a diferentes classes químicas em ervas medicinais e produtos acabados ${ }^{[52-55]}$.

\section{Considerações finais}

Ao longo dos anos, esse detector consolidou-se como uma importante ferramenta analítica na determinação de uma ampla variedade de compostos que não possuem cromóforos ou que tenham apenas grupos cromóforos fracos em suas estruturas químicas.

Os autores desta revisão buscam, com este artigo, contribuir para uma maior divulgação sobre o uso do detector evaporativo com espalhamento de luz (ELSD) acoplado à cromatografia líquida de alta eficiência.

\section{Agradecimentos}

Os autores agradecem o CNPq, a CAPES e a FAPESP pelo suporte financeiro e bolsas concedidas.

\section{Referências}

1 Vervoort N, Daemen D, Török G. Performance evaluation of evaporative light scattering detection and charged aerosol detection in reversed phase liquid chromatography. Journal of Chromatography 
A 2008; 1189:92-100. PMid:18054946. http://dx.doi. org/10.1016/j.chroma.2007.10.111

2 Charlesworth JM. Evaporative analyzer as a mass detector for liquid chromatography. Analytical Chemistry 1978; 50:1414-1420. http://dx.doi. org/10.1021/ac50033a011

3 Kohler M, Haredi W, Christen P, Veuthey JL. The evaporative light scattering detector: some applications in pharmaceutical analysis. Trends in Analytical Chemistry 1997; 16:475-484. http://dx.doi. org/10.1016/S0165-9936(97)00072-1

4 Ganzera M, Stuppner H. Evaporative light scattering detection (ELSD) for the analysis of natural products. Current Pharmaceutical Analysis 2005; 1:135-144. http://dx.doi.org/10.2174/1573412054022699

5 Megoulas NC, Koupparis MA. Twenty years of evaporative light scattering detection. Critical Reviews in Analytical Chemistry 2005; 35:301-316. http:// dx.doi.org/10.1080/10408340500431306

6 Mourey TH, Oppenheimer LE. Principles of operation of an evaporative light scattering detector for liquid chromatography. Analytical Chemistry 1984; 56:2427-2434. http://dx.doi. org/10.1021/ac00277a039

7 Righezza M, Guiochon G. Effects of the nature of the solvent and solutes on the response of a light scattering detector. Journal of Liquid Chromatography 1988; 11:1967-2004. http://dx.doi. org/10.1080/01483918808069036

8 Megoulas NC, Koupparis MA. Development and validation of a novel LC/ELSD method for the quantitation of gentamicin sulfate components in pharmaceuticals. Journal Pharmaceutical and Biomedical Analysis 2004; 36:73-79. PMid:15351050. http://dx.doi.org/10.1016/j.jpba.2004.05.018

9 Nogueira CM, Parmanhan BR, Farias PP, Corrêa AG. A importância crescente dos carboidratos em química medicinal. Revista Virtual de Química 2009; 1:149-159.

10 Stead DA. Current methodologies for the analysis of aminoglycosides. Journal of Chromatography B 2000; 747:69-93. http://dx.doi.org/10.1016/S03784347(00)00133-X

11 Nogueira LC, Silva F, Ferreira IMPLVO, Trugo LC. Separation and quantification of beer carboydrates by high-performance liquid chromatography with evaporative light scattering detection. Journal of ChromatographyA2005;1065:207-210.PMid:15782966. http://dx.doi.org/10.1016/j.chroma.2004.12.074

12 Pang $\mathrm{T}$ et al Determination of sugars in tobacco leaf by HPLC with evaporative light scattering detection. Journal Liquid Chromatography \& Related
Technologies 2006; 29:1281-1289. PMCid:1176066. http://dx.doi.org/10.1080/10826070600598993

13 Sharma U, Bhandari P, Kumar N, Singh B. Simultaneous determination of ten sugars in Tinospora cordifolia by ultrasonic assisted extraction and LC-ELSD. Chromatographia 2010; 71:633-638. http://dx.doi.org/10.1365/s10337-010-1520-2

14 Clarot I et al. Analysis of neomycin sulfate and framycetin sulfate by high-performance liquid chromatography using evaporative light scattering detection. Journal of Chromatography A 2005; 1087:236-244. PMid:16130719. http://dx.doi. org/10.1016/j.chroma.2005.05.054

15 Scheidl COR, Menzinger F, Maier EJ, Capek E, Scheidl O, Huck, CW. Simultaneous quantification of neomycin and bacitracin by LC-ELSD. Chromatographia 2009; 69:1-8. http://dx.doi. org/10.1365/s10337-009-1100-5

16 Clarot I, Storme-Paris I, Chaminade P, Estevenon O, Nicolas A, Rieutord A. Simultaneous quantitation of tobramycin and colistin sulphate by HPLC with evaporative light scattering detection. Journal of Pharmaceutical and Biomedical Analysis 2009; 50:64-67. PMid:19372021. http:// dx.doi.org/10.1016/j.jpba.2009.03.013

17 Megoulas NC, Koupparis MA. Direct determination of kanamycin in raw materials, veterinary formulation and culture media using a novel liquid chromatography-evaporative light scattering method. Analytica Chimica Acta 2005; 547:64-72. http://dx.doi. org/10.1016/j.aca.2004.11.031

18 Wang J, Hu X, Tu Y, Ni K. Determination of spectinomycin hydrochloride and its related substances by HPLC-ELSD and HPLC-MS ${ }^{\mathrm{n}}$. Journal of Chromatography B2006; 834:178-182. PMid:16545624. http://dx.doi.org/10.1016/j.jchromb.2006.02.045

19 Galanakis EG, Megoulas NC, Solich P, Koupparis MA. Development and validation of a novel LC non-derivatization method for the determination of amikacin in pharmaceuticals based on evaporative light scattering detection. Journal of Pharmaceutical and Biomedical Analysis 2006; 40:1114-1120. PMid:16242884. http://dx.doi.org/10.1016/j.jpba.2005.09.008

20 Sarri AK, Megoulas NC, Koupparis MA. Development of a novel method based on liquid chromatography-evaporative light scattering detection for the direct determination of streptomycin and dihydrostreptomycin in raw materials, pharmaceutical formulations, culture media and plasma. Journal of Chromatography A 2006; 1122:275-278. PMid:16806244. http://dx.doi.org/10.1016/j.chroma.2006.06.001 
21 Wang J, Hu X. Determination of arbekacin sulfate injection and its related substances by HPLC using evaporative light scattering detection. Journal of Liquid Chromatography \& Related Technologies 2010; 33:441-451. PMCid:1176066. http://dx.doi.org/10.1080/10826070903574170

22 Peterson BL, Cummings BS. A review of chromatographic methods for the assessment of phospholipids in biological samples. Biomedical Chromatography 2006; 20:227-243. PMid:16138296. http://dx.doi.org/10.1002/bmc.563

23 Cunha SC, Oliveira MBPP. Discrimination of vegetable oils by triacylglycerols evaluation of profile using HPLCELSD. Food Chemistry 2006; 95:518-524. http://dx.doi.org/10.1016/j.foodchem.2005.03.029

24 Park YW, Chang PS, Lee J. Application of triacylglycerol and fatty acid analyses to discriminate blended sesame oil with soybean oil. Food Chemistry 2010; 123:377-383. http://dx.doi. org/10.1016/j.foodchem.2010.04.049

25 Wang D et al. Determination of intramuscular phospholipid classes and molecular species in Gaoyou duck. Food Chemistry 2009; 112:150-155. http:// dx.doi.org/10.1016/j.foodchem.2008.05.052

26 Rodríguez-Alcalá LM, Fontecha J. Major lipid classes separation of buttermilk, and cows, goats and ewes milk by high performance liquid chromatography with an evaporative light scattering detector focused on the phospholipid fraction. Journal of Chromatography A 2010; 1217:3063-3066. PMid:20356599. http:// dx.doi.org/10.1016/j.chroma.2010.02.073

27 Duan J, Sugawara T, Hirata T. Rapid quantitative analysis of sphingolipids in seafood using HPLC with evaporative light scattering detection: its application in tissue distribution of sphingolipids in fish. Journal of Oleo Science 2010; 59:509-513. PMid:20720382. http://dx.doi.org/10.5650/jos.59.509

28 Torres CF, Vázquez L, Señoráns FJ, Reglero G. Study of the analysis of alkoxyglycerols and other non-polar lipids by liquid chromatography coupled with evaporative light scattering detector. Journal of Chromatography A 2005; 1078:28-34. PMid:16007978. http://dx.doi.org/10.1016/j.chroma.2005.04.015

29 Graeve M, Janssen D. Improved separation and quantification ofneutraland polarlipidclassesbyHPLCELSD using a monolithic silica phase: application to excepcional marine lipids. Journal of Chromatography B 2009; 877:1815-1819. PMid:19493709. http://dx.doi.org/10.1016/j.jchromb.2009.05.004

30 Nair VDP, Kanfer I, Hoogmartens J. Determination of stigmasterol, $\beta$-sitosterol and stigmastanol in oral dosage forms using high performance liquid chromatography with evaporative light scattering detection. Journal of Pharmaceutical and Biomedical Analysis 2006; 41:731-737. PMid:16487675. http:// dx.doi.org/10.1016/j.jpba.2005.12.030

31 Cong Y, Zhou YB, Chen J, Zeng YM, Wang JH. Alkaloid profiling of crude and processed Veratrum nigrum L. through simultaneous determination of ten steroidal alkaloids by HPLCELSD. Journal of Pharmaceutical and Biomedical Analysis 2008; 48:573-578. PMid:18599245. http://dx.doi.org/10.1016/j.jpba.2008.05.023

32 Wang L, Zhao D, Liu Y. Quality assessment of Veratrum nigrum L. by LC-ELSD fingerprints and LC quantitative analysis. Chromatographia 2008; 68:961-967. http:// dx.doi.org/10.1365/s10337-008-0799-8

33 Tang J et al. Simultaneous determination of six steroidal alkaloids of Veratrum dahuricum by HPLC-ELSD and HPLC-MS ${ }^{n}$. Chromatographia 2008; 67:15-21. http:// dx.doi.org/10.1365/s10337-007-0439-8

34 Oleszek W, Bialy Z. Chromatographic determination of plant saponins - an update (2002-2005). Journal of Chromatography A 2006; 1112:78-91. PMid:16451803. http://dx.doi.org/10.1016/j.chroma.2006.01.037

35 Fuzzati N. Analysis methods of ginsenosides. Journal of Chromatography B 2004; 812:119-133. PMid:15556492

36 Sun BS et al. Simultaneous quantification of 19 ginsenosides in black ginseng developed from Panax ginseng by HPLC-ELSD. Journal of Pharmaceutical and Biomedical Analysis 2009; 50:15-22. PMid:19394786. http://dx.doi.org/10.1016/j.jpba.2009.03.025

37 Lee KY et al. Quality control of Pulsatilla koreana based on the simultaneous determination of triterpenoidal saponins by HPLC-ELSD and principal component analysis. Phytochemical Analysis 2010; 21:314-321. PMid:20066731. http://dx.doi.org/10.1002/pca.1201

38 Eom HY et al. Comparison between evaporative light scattering detection and charged aerosol detection for theanalysisofsaikosaponins. JournalofChromatography A 2010; 1217:4347-4354. PMid:20452602. http://dx.doi.org/10.1016/j.chroma.2010.04.047

39 Sun F, He Q, Xiao P, Muhammad I, Cheng Y. Simultaneous quantification of five triterpenoid saponins in Clematis L. Spp. by high-performance liquid chromatography with evaporativelightscattering detection. Phytochemical Analysis 2008; 19:40-45. PMid:17654533. http://dx.doi.org/10.1002/pca.1013

40 Man S et al. Qualitative and quantitative determination of major saponins in Paris and Trillium by HPLCELSD and HPLC-MS/MS. Journal of Chromatography 
B 2010; 878:2943-2948. PMid:20870470. http://dx.doi. org/10.1016/j.jchromb.2010.08.033

41 Liu XX, Wang L, Yang J, Zhang TT, Deng XD, Wang Q. Simultaneous analysis of eight bioactive steroidal saponins in Gongxuening capsules by HPLC-ELSD and HPLC-MS ${ }^{n}$. Acta Chromatographica 2009; 21:327-339. http://dx.doi.org/10.1556/AChrom.21.2009.2.11

42 van Beek TA, Montoro P. Chemical analysis and quality control of Ginkgo biloba leaves, extracts, and phytopharmaceuticals. Journal of Chromatography A 2009; 1216:2002-2032. PMid:19195661. http:// dx.doi.org/10.1016/j.chroma.2009.01.013

43 Celeghini RMS, Souza IMO, Silva AP, Rodrigues AF, Foglio MA. Desenvolvimento e validação de metodologia analítica por CLAE-IR para determinação de artemisinina em Artemisia annua L. Química Nova 2009; 32:875-878. http://dx.doi. org/10.1590/S0100-40422009000400009

44 Luo XL, Shao Q, Qu HB, Cheng YY. Simple method for determination of five terpenoids from different parts of Tripterygium wilfordii and its preparations by HPLC coupled with evaporative light scattering detection. Journal of Separation Science 2007; 30:1284-1291. PMid:17623469. http://dx.doi.org/10.1002/jssc.200600450

45 Chen XQ et al. Quantitative analysis of triterpenoids in different parts of Ilex hainanensis, Ilex stewardii and Ilex pubescens using HPLC-ELSD and HPLC-MS ${ }^{\mathrm{n}}$ and antibacterial activity. Food Chemistry 2011; 126:1454-1459. http://dx.doi. org/10.1016/j.foodchem.2010.11.136

46 Kaur P, Chaudhary A, Singh B, Gopichand RD. Optimization of extraction technique and validation of developed RP-HPLC-ELSD method for determination of terpene trilactones in Ginkgo biloba leaves. Journal of Pharmaceutical and Biomedical Analysis 2009; 50:1060-1064. PMid:19616398. http:// dx.doi.org/10.1016/j.jpba.2009.06.036

47 Dubber MJ, Kanfer I. Determination of terpene trilactones in Ginkgo biloba solid oral dosage forms using HPLC with evaporative light scattering detection. Journal of Pharmaceutical and Biomedical Analysis 2006; 41:135-140. PMid:16406712. http:// dx.doi.org/10.1016/j.jpba.2005.11.010

48 Avula B et al. Comparison of LC-UV, LC-ELSD and LC-MS methods for the determination of sesquiterpenoids in various species of Artemisia. Chromatographia 2009; 70:797-803. http://dx.doi. org/10.1365/s10337-009-1237-2

49 Lapkin AA, Walker A, Sullivan N, Khambay B, Mlambo B, Chemat S. Development of HPLC analytical protocols for quantification of artemisinin in biomass and extracts. Journal of Pharmaceutical and Biomedical Analysis 2009; 49:908-915. PMid:19231128. http:// dx.doi.org/10.1016/j.jpba.2009.01.025

50 Wang S, Yang FQ, Feng K, Li DQ, Zhao J, Li SP. Simultaneous determination of nucleosides, myriocin, and carbohydrates in Cordyceps by HPLC coupled with diode array detection and evaporative light scattering detection. Journal of Separation Science 2009; 32:4069-4076. PMid:20066678. http:// dx.doi.org/10.1002/jssc.200900570

51 Avula B et al Quantitative determination of flavonoids and cycloartanol glycosides from aerial parts of Sutherlandia frutescens (L.) R. BR. by LC-UV/ ELSD methods and confirmation by using LC-MS method. Journal of Pharmaceutical and Biomedical Analysis 2010; 52:173-180. PMid:20122811. http:// dx.doi.org/10.1016/j.jpba.2010.01.010

52 Liu EH, Qi LW, Cheng XL, Peng YB, Li P. Simultaneous determination of twelve bioactive constituents in Buyang Huanwu decoction by HPLC-DAD-ELSD and HPLC-TOF/MS. Biomedical Chromatography 2010; 24:125-131. PMid:19630070

53 Qi LW et al. Simultaneous determination of 15 marker constituents in various radix Astragali preparations by solid-phase extraction and high-performance liquid chromatography. Journal of Separation Science 2008; 31:97-106. PMid:18069702. http:// dx.doi.org/10.1002/jssc.200700286

54 Yi L, Qi LW, Li P, Ma YH, Luo YJ, Li HY. Simultaneous determination of bioactive constituents in Danggui Buxue Tang for quality control by HPLC coupled with a diode array detector, an evaporative light scattering detector and mass spectrometry. Analytical and Bioanalytical Chemistry 2007; 389:571-580. PMid:17653535. http://dx.doi.org/10.1007/s00216007-1431-8

55 Wei YJ, Qi LW, Li P, Luo HW, Yi L, Sheng LH. Improved quality control method for Fufang Danshen preparations through simultaneous determination of phenolic acids, saponins and diterpenoid quinones by HPLC coupled with diode array and evaporative light scattering detectors. Journal of Pharmaceutical and Biomedical Analysis 2007; 45:775-789. PMid:17720349. http://dx.doi.org/10.1016/j.jpba.2007.07.013

Recebido: 21/09/201 1 Aceito: 18/10/2011 